Purdue University

Purdue e-Pubs

2017

\title{
Spatiotemporal Infrared Measurement of Interface Temperatures During Water Droplet Evaporation on a Nonwetting Substrate
}

\author{
A. Chandramohan \\ Purdue University \\ J.A. Weibel \\ Purdue University, jaweibel@purdue.edu \\ S V. Garimella \\ Purdue University, sureshg@purdue.edu
}

Follow this and additional works at: http://docs.lib.purdue.edu/coolingpubs

Chandramohan, A.; Weibel, J. A.; and Garimella, S V., "Spatiotemporal Infrared Measurement of Interface Temperatures During Water Droplet Evaporation on a Nonwetting Substrate" (2017). CTRC Research Publications. Paper 314.

http://dx.doi.org/http://dx.doi.org/10.1063/1.4975003

This document has been made available through Purdue e-Pubs, a service of the Purdue University Libraries. Please contact epubs@purdue.edu for additional information. 


\title{
Spatiotemporal infrared measurement of interface temperatures during water droplet evaporation on a nonwetting substrate
}

\author{
Aditya Chandramohan, Justin A. Weibel, and Suresh V. Garimella ${ }^{\text {a) }}$ \\ School of Mechanical Engineering, Purdue University, West Lafayette, Indiana 47907, USA
}

(Received 1 December 2016; accepted 11 January 2017; published online 25 January 2017)

\begin{abstract}
High-fidelity experimental characterization of sessile droplet evaporation is required to understand the interdependent physical mechanisms that drive the evaporation. In particular, cooling of the interface due to release of the latent heat of evaporation, which is not accounted for in simplified vapor-diffusion-based models of droplet evaporation, may significantly suppress the evaporation rate on nonwetting substrates, which support tall droplet shapes. This suppression is counteracted by convective mass transfer from the droplet to the air. While prior numerical modeling studies have identified the importance of these mechanisms, there is no direct experimental evidence of their influence on the interfacial temperature distribution. Infrared thermography is used here to simultaneously measure the droplet volume, contact angle, and spatially resolved interface temperatures for water droplets on a nonwetting substrate. The technique is calibrated and validated to quantify the temperature measurement accuracy; a correction is employed to account for reflections from the surroundings when imaging the evaporating droplets. Spatiotemporally resolved interface temperature data, obtained via infrared thermography measurements, allow for an improved prediction of the evaporation rate and can be utilized to monitor temperature-controlled processes in droplets for various lab-on-a-chip applications. Published by AIP Publishing.
\end{abstract}

[http://dx.doi.org/10.1063/1.4975003]

Droplet evaporation plays a defining role in DNA microarray manufacturing, ${ }^{1}$ droplet-based biosensors, ${ }^{2-4}$ droplet mixing, ${ }^{5}$ inkjet printing, ${ }^{6}$ and droplet-based particle deposition. ${ }^{7}$ In these applications, an understanding of the thermal and species transport characteristics is critical for controlling the droplet evaporation behavior and deposition of suspended particulates. The inspection of interface temperatures is crucial to understanding and controlling the thermal and evaporative behavior of droplets for various droplet-based manufacturing and testing applications.

Several measurement techniques have been applied for characterization of droplet temperatures, including microencapsulated thermochromic liquid crystals (TLCs), ${ }^{8}$ thermocouples,,${ }^{9,10}$ digital holographic interferometry,${ }^{11}$ and infrared thermography. ${ }^{12,13}$ Digital holographic interferometry can be used to calculate the water vapor concentration around a droplet and infer the interface temperature and local evaporation rate. Microencapsulated TLCs exhibit a temperaturedependent change in hue, which can be captured using a color camera. Both microencapsulated TLCs and thermocouples are intrusive and provide temperature measurements at discrete locations; the measurements provide temperatures close to the interface, but not at the interface itself. Infrared thermography yields a high-spatial-resolution temperature field; furthermore, it is entirely nonintrusive and does not require the complex system of lasers and mirrors needed for digital holographic interferometry. For organic liquids that are semi-transparent in the infrared spectrum, such as ethanol, methanol, and acetone, infrared thermography captures a temperature signature integrated over the penetration depth and has been used to observe convective patterns within

a)Telephone: (765) 494-5621. Electronic mail: sureshg@purdue.edu evaporating droplets. ${ }^{13-15}$ Water is virtually opaque in the mid-wavelength infrared spectrum, ${ }^{16}$ allowing for temperature measurements at the interface. Girard et al. ${ }^{17}$ applied this technique to measure the temperature of water droplets on a wetting substrate; Saha et al. ${ }^{18}$ measured the effect of laser heating on the evaporation of acoustically levitated droplets. While some instantaneous infrared temperature measurements have been conducted for droplets evaporating on nonwetting substrates, ${ }^{19}$ none have accounted for reflections from background radiation incident on the curved surface of the droplet. Coatings can be applied to the solid surface to remove such reflection effects, ${ }^{20}$ whereas this is not possible with liquid-air interfaces. Moreover, no studies have utilized infrared thermography visualizations to capture the temporal droplet (volume) evolution during evaporation alongside temperature measurements.

Accurate measurement of droplet interface temperatures during evaporation is required for improving and validating modeling efforts that have historically neglected important thermal transport mechanisms. Picknett and Bexon ${ }^{21}$ developed one of the earliest models for sessile droplet evaporation by applying Maxwell's diffusion-electrostatic potential analogy, ${ }^{22}$ while assuming uniform evaporative mass flux. Several later studies experimentally and numerically explored vapor diffusion around low-contact-angle (i.e., wetting) evaporating droplets and concluded that evaporative mass flux is nonuniform over the droplet surface. ${ }^{23-25}$ Popov $^{26}$ developed a closed-form, analytical solution of vapor species diffusion through air in the surrounding domain to predict the evaporation of droplets exhibiting any contact angle; however, a key assumption in the boundary conditions is that the droplet surface is at a uniform temperature, which inherently neglects the influence of thermal transport within the droplet 
itself. Several subsequent studies have demonstrated that convective heat and mass transfer to the surroundings can be significant. $^{27-30}$

An experimental study by Dash and Garimella ${ }^{31}$ revealed a significant discrepancy between vapor-diffusionbased model predictions and the measured rate of droplet evaporation on nonwetting surfaces, which was attributed to a large temperature drop along the droplet height induced by evaporative cooling. High-fidelity numerical modeling by Pan and coworkers ${ }^{27,32}$ mapped the competing effects of external natural convection and evaporative cooling as a function of the surface wettability; the relatively tall droplets supported on nonwetting surfaces have a large effective thermal resistance between the substrate and droplet interface, such that evaporative cooling governs the droplet temperature profile and evaporation rate. Gleason and Putnam ${ }^{33}$ showed that imposing a nonuniform interface temperature profile as a correction to the vapor-diffusion model more accurately predicted the experimental evaporation data. It is clear that spatially resolved temperature measurements are needed to capture the evaporation characteristics throughout the droplet. This letter demonstrates calibrated infrared measurement of the temperature distribution on the surface of droplets evaporating on nonwetting substrates over a range of temperatures. The temporal evolution of droplet volume, contact angle, temperature profiles along the droplet height, and the evaporation rate are simultaneously tracked.

The nonwetting copper substrate used in this study was fabricated in the Birck Nanotechnology Center at Purdue University. The substrate was immersed in an aqueous solution of $2 \mathrm{M} \mathrm{NaOH}$ and $0.1\left(\mathrm{NH}_{4}\right)_{2} \mathrm{~S}_{2} \mathrm{O}_{8}$ for 60 min to etch copper oxide nanostructures. It was then rinsed with deionized water and dried with $\mathrm{N}_{2}$ gas. To attain superhydrophobicity, the nanostructured surface was immersed in a $0.001 \mathrm{M} \mathrm{n}$ hexane solution of $1 \mathrm{H}, 1 \mathrm{H}, 2 \mathrm{H}, 2 \mathrm{H}$-perfluorooctyltrichlorosilane (PFOS) for $60 \mathrm{~min}$ followed by heat treatment on a hotplate at $\sim 150{ }^{\circ} \mathrm{C}$ for $60 \mathrm{~min}$. A localized surface indentation is introduced to provide a consistent location where the droplet pins to the substrate to allow for repeatable visualization.

The experimental facility used to capture the infrared images of evaporating droplets is shown in Figure 1. The substrate is held at a fixed temperature by attaching it to an insulated copper block that is heated from the bottom by a

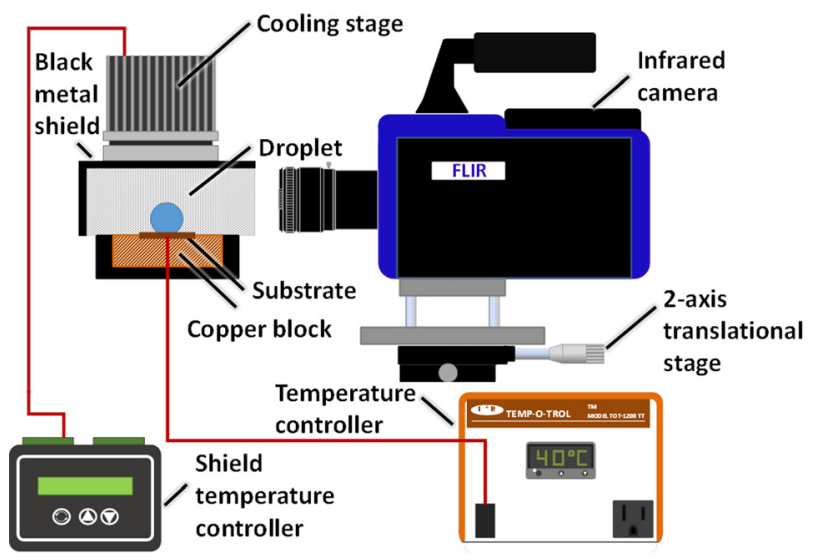

FIG. 1. Schematic diagram of the experimental facility. polyimide heater with a feedback temperature controller (TOT-1200, Temp-O-Trol). In order to maintain consistent background radiation, a black-painted (ColorMaster ${ }^{\mathrm{TM}}$ Flat Black, Krylon; emissivity of 0.97) aluminum shield surrounds the droplet. The temperature of the metal shield is maintained using a temperature-controlled thermoelectric cooling stage (CP-031HT, TE Technology, Inc.). All experiments were conducted with the shield held at $20 \pm 0.1^{\circ} \mathrm{C}$. The ambient relative humidity was $28 \pm 3 \%$.

The temperature of the droplet surface is measured with an infrared camera (SC7650, FLIR). A $50 \mathrm{~mm}$ lens (Nyctea, Janos) that is fitted with $30 \mathrm{~mm}$ of extension tubes yields a magnification of $\sim 0.76$. This lens and camera system captures radiation in the mid-wavelength infrared range $(1.5 \mu \mathrm{m}$ to $5.1 \mu \mathrm{m})$, which coincides with the peak emission wavelength (viz., $3 \mu \mathrm{m}$ ) of water. ${ }^{16}$ Further details on the blackbody calibration, uncertainty assessment, and validation of infrared temperature measurement of water-air interfaces are included in the supplementary material. The uncertainty of the infrared temperature measurement is $0.41{ }^{\circ} \mathrm{C}$. The camera records frames at $5 \mathrm{~Hz}$.

To perform an experiment, a water droplet of approximately $4 \mu \mathrm{l}$ is deposited on the heated substrate using a pipette (AP-10, AccuPet). At the volumes used, the effects of gravity on the interface curvature can be neglected and the droplet assumes a spherical cap geometry. The droplet is allowed time to settle after being placed on the surface; temperature data are only presented from the time the droplet volume has reduced to $3 \mu \mathrm{l}$ until complete evaporation. Five repeated droplet evaporation trials are performed at each of the four different fixed substrate temperatures: $30 \pm 0.5^{\circ} \mathrm{C}$, $40 \pm 0.5^{\circ} \mathrm{C}, 50 \pm 0.5^{\circ} \mathrm{C}$, and $60 \pm 0.5^{\circ} \mathrm{C}$.

The infrared visualizations of the droplet are used to simultaneously track the droplet temperature and shape throughout the evaporation process, as shown in Figure 2. Sample videos at each substrate temperature are shown in the supplementary material. The droplet contact diameter and height are measured at each frame and used to calculate the droplet volume and contact angle based on geometric relations for a spherical cap shape. The volume and contact angle evolution of the droplets are plotted with respect to time in Figure 3 at intervals of $\sim 30 \mathrm{~s}$. As shown in Figure 3(a), the volume decreases in a similar, exponential trend to those reported in the literature. ${ }^{5,31}$ The evaporation rate increases significantly with increasing substrate temperature. The contact line remains pinned and the evaporation primarily follows a constant-contact radius mode; as shown in Figure 3(b), the contact radius is nearly constant during the course of evaporation. The contact angle continuously decreases en route to complete evaporation.

The uncorrected interface temperatures shown in Figure 2 include the effects of background radiation. To proceed with further quantitative analysis, the raw interface temperature data acquired with infrared thermography must be corrected to account for the reflection of background radiation off the droplet to the camera sensor. Assuming that the incident radiation on the droplet interface is unpolarized, the specular reflectivity can be calculated using the Fresnel equations. ${ }^{34}$ Due to the spherical shape of the droplet, the reflectivity of the droplet changes as a function of the angle 
(a)

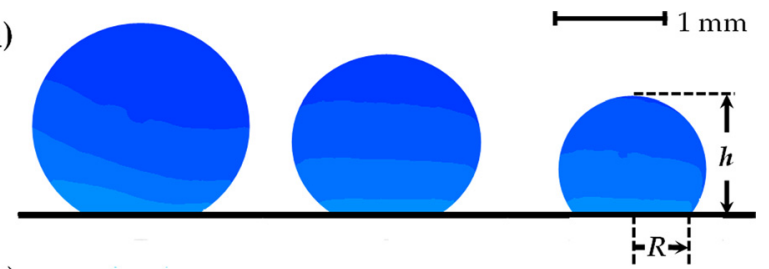

(b)

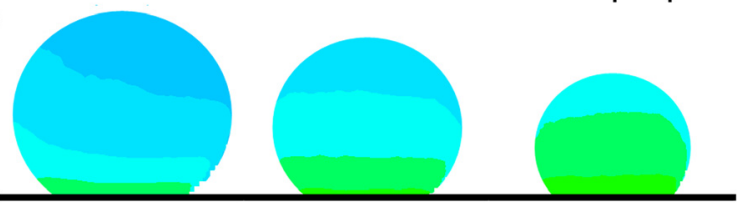

(c)

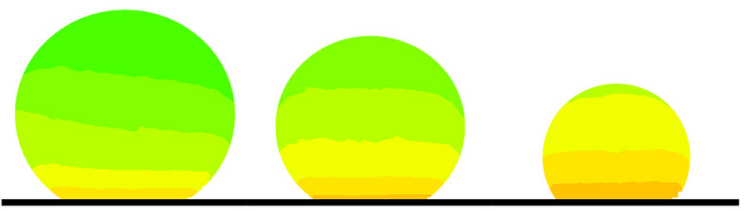

(d)

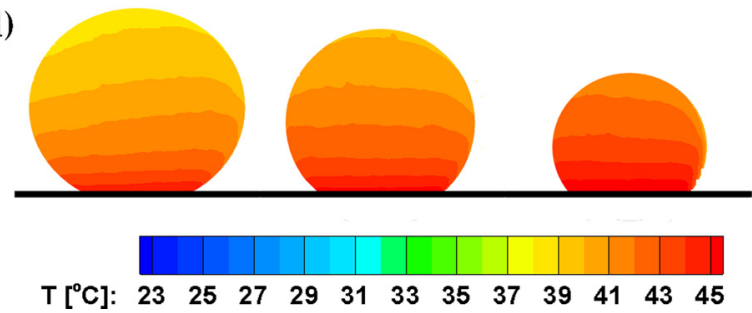

FIG. 2. The uncorrected infrared temperature data are shown for droplet evaporation trials at substrate temperatures of (a) $30^{\circ} \mathrm{C}$, (b) $40^{\circ} \mathrm{C}$, (c) $50^{\circ} \mathrm{C}$, and (d) $60^{\circ} \mathrm{C}$; from left to right, the panels show droplet volumes of $3 \mu$, $2 \mu \mathrm{l}$, and $1 \mu \mathrm{l}$. The contact radius, $R$, and the droplet height, $h$, are marked for the top right case.

between the surface normal of the droplet and the image plane. Hence, the droplet shape parameters are used to locally correct for the reflection for the entire droplet surface. Details of the reflection-correction procedure are presented in the supplementary material.

All the infrared temperature data are corrected using this procedure; an example interface temperature contour of a droplet on a $50^{\circ} \mathrm{C}$ substrate is shown in Figure 4. A complete set of corrected infrared temperature data for each substrate temperature and a range of droplet volumes are included in the supplementary material. As can be seen in Figure 4, evaporative cooling has a significant effect on the droplet temperature distribution, resulting in a large temperature gradient along the height of the droplet. The resistance to thermal transport from the substrate to the top of the droplet leads to a significant temperature drop at the top of the

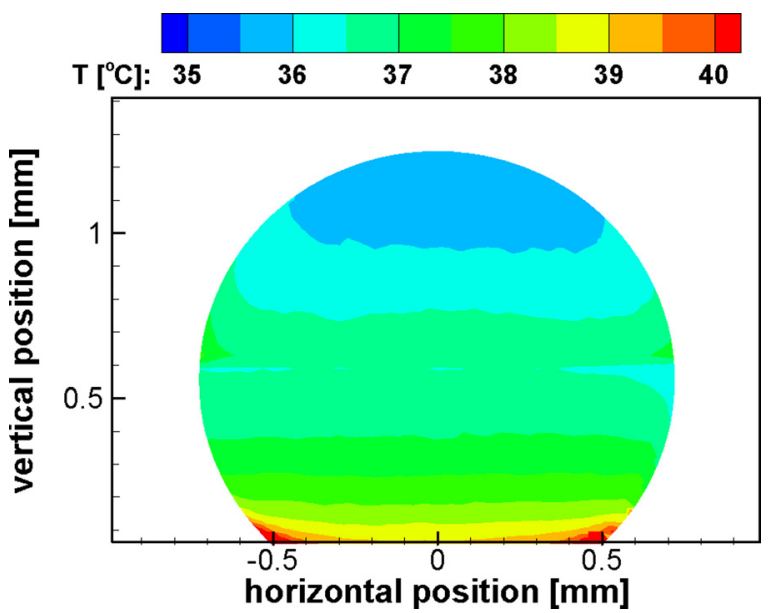

FIG. 4. Temperature data after correcting for background reflections for a $2 \mu \mathrm{l}$ droplet evaporating on a substrate at a temperature of $50^{\circ} \mathrm{C}$.

droplet due to evaporative cooling. We note that the maximum interface temperature measured near the bottom of the droplet $\left(\sim 39^{\circ} \mathrm{C}\right)$ is lower than the nominal bulk substrate temperature $\left(50^{\circ} \mathrm{C}\right)$ due to localized evaporative cooling of the substrate directly beneath the droplet. A similar localized cooling effect was reported by Gleason et al. ${ }^{35}$ based on infrared measurements of the substrate temperature in the presence of an evaporating droplet. The net evaporation rate from the droplet is determined by this non-uniform droplet interface temperature distribution; this effect is ignored in simplified vapor-diffusion-based models ${ }^{26}$ that assume a uniform temperature for the entire interface. As a measure of the evaporative cooling effect, the temperature drop across the height of the droplet is plotted as a function of the droplet volume in Figure 5. The temperature drop decreases as the droplet volume decreases with progressive evaporation. The reduction in the droplet height and contact angle with evaporation reduces the thermal transport resistance from the substrate to the interface, thus homogenizing the droplet temperature. This is consistent with high-fidelity modeling efforts that have shown a decreasing influence of evaporative cooling with decreasing droplet height-to-contact-diameter aspect ratio. ${ }^{32}$

The experimental evaporation rate shown in Figure 6 is calculated based on the derivative of droplet volume with time from the data shown in Figure 3. The evaporation rate decreases with decreasing volume during the course of evaporation. Even though the evaporation flux on the interface increases with decreasing volume (particularly drastically at the contact line), ${ }^{31}$ the surface area of the droplet available
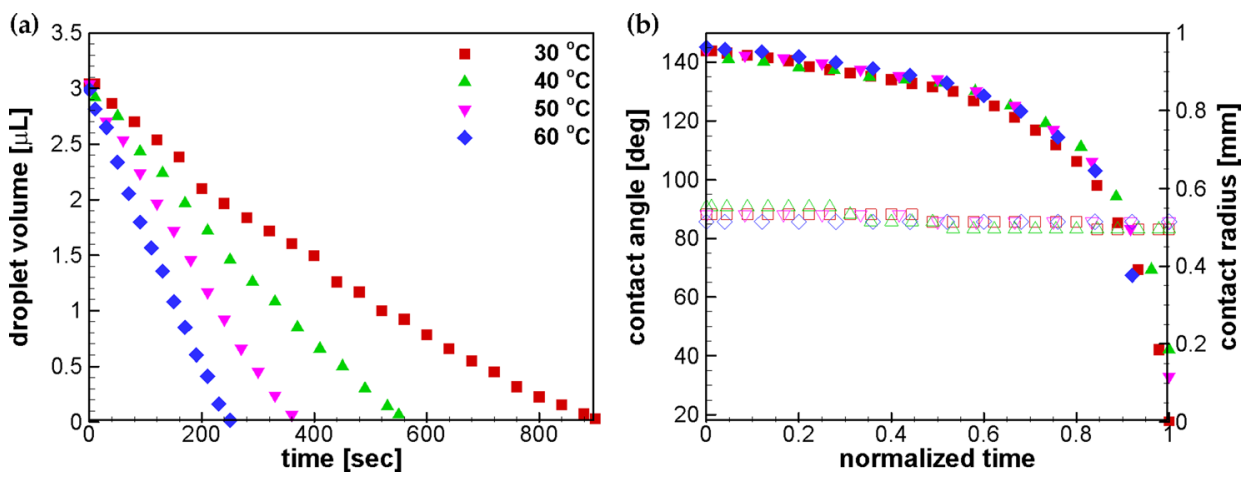

FIG. 3. (a) The volume and (b) contact angle (solid symbols) and contact radius (hollow symbols) of the droplet are shown as a function of time for selected droplet evaporation trials at each substrate temperature. The time axis in (b) is normalized by the total evaporation time in each case. 


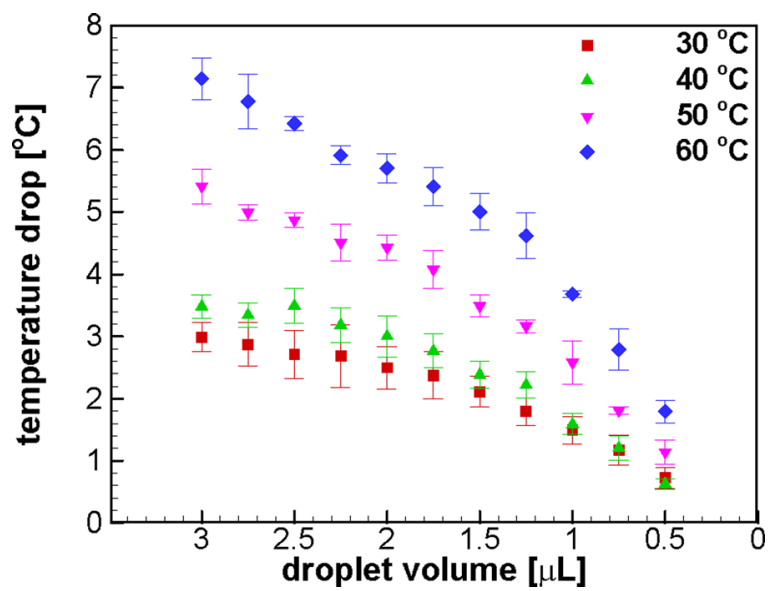

FIG. 5. The temperature drop across the height of the droplet is plotted as a function of droplet volume (at intervals of $0.25 \mu \mathrm{l}$ ) for all substrate temperatures. The error bars represent the standard deviations across all trials at that substrate temperature.

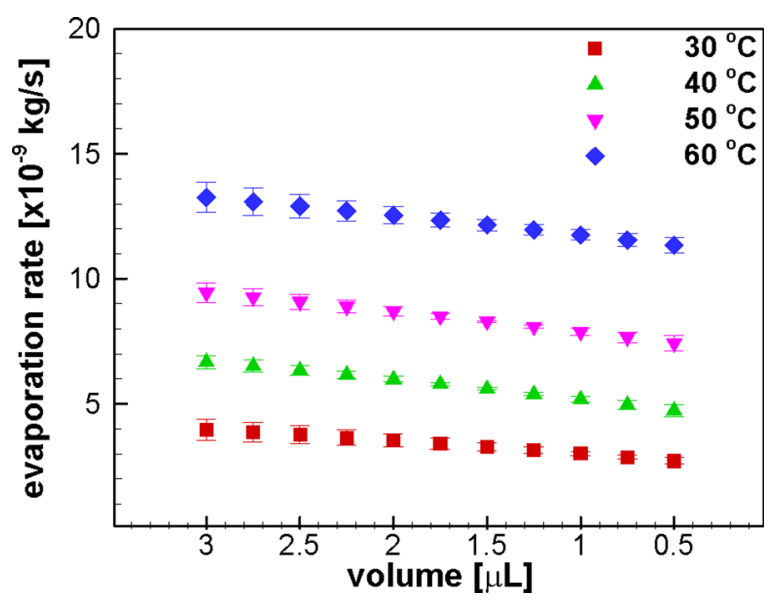

FIG. 6. The experimental evaporation rate plotted as a function of droplet volume (at intervals of $0.5 \mu \mathrm{l}$ ) for all substrate temperatures. The error bars represent the standard deviation across all trials at a given substrate temperature.

for evaporation decreases sufficiently such that the net total rate reduces. Further analysis in the supplementary material incorporates the interface temperature data into vapor-diffusion-based and natural-convection-based predictions of the evaporation rate, to assess the relative importance of these transport mechanisms for the current data.

Infrared thermography is exploited to capture the spatiotemporal interface temperature distribution during evaporation of a droplet on a nonwetting substrate; the volume and shape evolution of the droplet are tracked simultaneously with the interface temperature. This approach meets the need for accurate, localized temperature data to characterize salient features of droplet evaporation, as called for in past studies. ${ }^{33,36}$ In addition, such data are critical to applications in which the droplet temperatures control physical processes other than evaporation, such as voltage-induced modulation of droplet temperatures for biosensing, ${ }^{37}$ control of reaction rates in droplet microfluidics, ${ }^{38,39}$ and temperature modulation to denature DNA for polymerase chain reactions. ${ }^{40,41}$ The methodology is also broadly applicable for characterizing the temperature of curved interfaces.
See supplementary material for calibration and validation of infrared measurement, videos of the experimental infrared measurements, infrared measurement corrections, corrected temperature data, and the interface-temperaturebased evaporation rate prediction.

Special thanks to Dr. Xuemei Chen (Purdue University) for fabricating the substrates used for these experiments.

${ }^{1}$ V. Dugas, J. Broutin, and E. Souteyrand, Langmuir 21, 9130 (2005).

${ }^{2}$ F. D. Angelis, F. Gentile, F. Mecarini, G. Das, M. Moretti, P. Candeloro, M. L. Coluccio, G. Cojoc, A. Accardo, C. Liberale, R. P. Zaccaria, G. Perozziello, L. Tirinato, A. Toma, G. Cuda, R. Cingolani, and E. D. Fabrizio, Nat. Photonics 5, 682 (2011).

${ }^{3}$ A. Ebrahimi, P. Dak, E. Salm, S. Dash, S. V. Garimella, R. Bashir, and M. A. Alam, Lab Chip 13, 4248 (2013).

${ }^{4}$ P. Dak, A. Ebrahimi, V. Swaminathan, C. Duarte-Guevara, R. Bashir, and M. A. Alam, Biosensors 6, 14 (2016).

${ }^{5}$ S. Dash, A. Chandramohan, J. A. Weibel, and S. V. Garimella, Phys. Rev. E 90, 62407 (2014).

${ }^{6}$ D. Soltman and V. Subramanian, Langmuir 24, 2224 (2008).

${ }^{7}$ M. Dicuangco, S. Dash, J. A. Weibel, and S. V. Garimella, Appl. Phys. Lett. 104, 201604 (2014).

${ }^{8}$ C. D. Richards and R. F. Richards, Exp. Fluids 25, 392 (1998).

${ }^{9}$ S. David, K. Sefiane, and L. Tadrist, Colloids Surf., A 298, 108 (2007).

${ }^{10}$ A. Chandramohan, S. Dash, J. A. Weibel, X. Chen, and S. V. Garimella, Langmuir 32, 4729 (2016).

${ }^{11}$ S. Dehaeck, A. Rednikov, and P. Colinet, Langmuir 30, 2002 (2014).

${ }^{12}$ G. Fabien, M. Antoni, and K. Sefiane, Langmuir 27, 6744 (2011).

${ }^{13}$ B. Sobac and D. Brutin, Phys. Rev. E 86, 21602 (2012).

${ }^{14}$ K. Sefiane, J. R. Moffat, O. K. Matar, and R. V. Craster, Appl. Phys. Lett. 93, 74103 (2008).

${ }^{15}$ K. Sefiane, Y. Fukatani, Y. Takata, and J. Kim, Langmuir 29, 9750 (2013).

${ }^{16}$ W. M. Irvine and J. B. Pollack, Icarus 8, 324 (1968).

${ }^{17}$ F. Girard, M. Antoni, and K. Sefiane, Langmuir 26, 4576 (2010).

${ }^{18}$ A. Saha, S. Basu, C. Suryanarayana, and R. Kumar, Int. J. Heat Mass Transfer 53, 5663 (2010).

${ }^{19}$ D. Tam, V. von Arnim, G. H. McKinley, and A. E. Hosoi, J. Fluid Mech. 624, 101 (2009).

${ }^{20}$ C. Meola and G. M. Carlomagno, Meas. Sci. Technol. 15, R27 (2004).

${ }^{21}$ R. G. Picknett and R. Bexon, J. Colloid Interface Sci. 61, 336 (1977).

${ }^{22}$ J. C. Maxwell, The Scientific Papers of James Clerk Maxwell (Cambridge University Press, 1890), p. 625.

${ }^{23}$ R. D. Deegan, O. Bakajin, T. F. Dupont, G. Huber, S. R. Nagel, and T. A. Witten, Nature 389, 827 (1997).

${ }^{24}$ R. D. Deegan, O. Bakajin, T. F. Dupont, G. Huber, S. R. Nagel, and T. A. Witten, Phys. Rev. E 62, 756 (2000).

${ }^{25}$ H. Hu and R. G. Larson, J. Phys. Chem. B 106, 1334 (2002).

${ }^{26}$ Y. O. Popov, Phys. Rev. E 71, 36313 (2005).

${ }^{27}$ Z. Pan, S. Dash, J. A. Weibel, and S. V. Garimella, Langmuir 29, 15831 (2013).

${ }^{28}$ M. Ait Saada, S. Chikh, and L. Tadrist, Phys. Fluids 22, 112115 (2010).

${ }^{29}$ Z. Zheng, L. Zhou, X. Du, and Y. Yang, Int. J. Heat Mass Transfer 101, 10 (2016).

${ }^{30}$ F. Carle, S. Semenov, M. Medale, and D. Brutin, Int. J. Therm. Sci. 101, 35 (2016).

${ }^{31}$ S. Dash and S. V. Garimella, Langmuir 29, 10785 (2013).

${ }^{32}$ Z. Pan, J. A. Weibel, and S. V. Garimella, Langmuir 30, 9726 (2014).

${ }^{33}$ K. Gleason and S. A. Putnam, Langmuir 30, 10548 (2014).

${ }^{34}$ M. F. Modest, Radiative Heat Transfer (Academic Press, 2013).

${ }^{35}$ K. Gleason, H. Voota, and S. A. Putnam, Int. J. Heat Mass Transfer 101, 418 (2016).

${ }^{36}$ S. Dash and S. V. Garimella, Phys. Rev. E 89, 42402 (2014).

${ }^{37}$ E. Salm, C. D. Guevara, P. Dak, B. R. Dorvel, B. Reddy, M. A. Alam, and R. Bashir, Proc. Natl. Acad. Sci. 110, 3310 (2013).

${ }^{38}$ B. Ahmed, D. Barrow, and T. Wirth, Adv. Synth. Catal. 348, 1043 (2006).

${ }^{39}$ S.-Y. Teh, R. Lin, L.-H. Hung, and A. P. Lee, Lab Chip 8, 198 (2008).

${ }^{40}$ H. Kim, S. Vishniakou, and G. W. Faris, Lab Chip 9, 1230 (2009).

${ }^{41}$ Y. Zhang, Y. Zhu, B. Yao, and Q. Fang, Lab Chip 11, 1545 (2011). 